\title{
COMBINATORICS AND TOPOLOGY OF LINE ARRANGEMENTS IN THE COMPLEX PROJECTIVE PLANE
}

\author{
ENRIQUE ARTAL-BARTOLO
}

(Communicated by Ronald Stern)

\begin{abstract}
We use some results about Betti numbers of coverings of complements of plane projective curves to discuss the problem of how combinatorics determine the topology of line arrangement, finding a counterexample to a conjecture of Orlik.
\end{abstract}

\section{INTRODUCTION}

In [E], a formula for Betti numbers of the Milnor fiber of the cone over a projective plane curve is given involving the dual of some differentials which arise in a spectral sequence. We have presented this formula in a more precise form in [A], showing an explicit relation with the infinitely near points. In this paper we are going to apply these results to the theory of line arrangements in the complex projective plane $\mathbb{P}^{2}$.

Let $f \in \mathbb{C}[X, Y, Z]$ be a reduced homogeneous polynomial of degree $N$ and $\mathbf{C}:=\left\{[x: y: z] \in \mathbb{P}^{2} \mid f(x, y, z)=0\right\}$ the curve defined by $f$; let $r$ be the number of global irreducible components. Let $E:=\mathbb{P}^{2} \backslash \mathbf{C}$, be the complement of the curve and let $X:=\left\{(x, y, z) \in \mathbb{C}^{3}: f(x, y, z)=1\right\} ; X$ is the Milnor fiber of the singularity defined by $f$, namely, the cone over $\mathbf{C}$. The projection $\tau: X \rightarrow E$, such that $\tau(x, y, z):=[x: y: z]$, is an $N$-fold cyclic covering. Its automorphism group is $\mu_{N}$, the group of the $N$ th roots of unity in $\mathbb{C}$; an element $\zeta \in \mu_{N}$ acts by $\zeta \cdot(x, y, z)=(\zeta x, \zeta y, \zeta z)$.

We are going to recall the formula for $b_{1}(X)$ in terms of the position of singularities of $\mathbf{C}$ and some annihilation theorems from $[\mathrm{Z} 1, \mathrm{E}]$.

In the last section we establish another proof of the nonexistence of curves of degree 7 (resp. 8) with 11 (resp. 16) cusps; these results come from [Z2]. We end with some applications to the theory of hyperplane arrangements. We study the next conjecture, communicated to the author by P. Orlik.

1.1. Conjecture. Let $\mathbf{C}$ be a reduced projective plane curve of degree $N$ whose irreducible components are lines. Let $\zeta: X \rightarrow X$ be a generator of the automorphism group of the covering $\tau: X \rightarrow E$. Then $\zeta^{*}: H^{1}(X, \mathbb{C}) \rightarrow H^{1}(X, \mathbb{C})$ is the identity.

Received by the editors September 28, 1992.

1991 Mathematics Subject Classification. Primary 14N10, 14F45; Secondary 14F25, 32 S55.

Key words and phrases. Singularity, Milnor fiber, combinatorics, Betti numbers, arrangements. 
1.2. Remark. The quotient of $H^{*}(X, \mathbb{C})$ by the action of $\zeta^{*}$ is identified with the complex cohomology of the quotient of $X$ by the action of $\zeta$. This quotient is the base $E$ of the covering. Then, this conjecture is equivalent to

$$
b_{1}(X)=b_{1}(E), \quad b_{2}(X)=b_{2}(E)+(N-1) \chi(E) .
$$

We find counterexamples to this conjecture.

2. THE FORMULA FOR $b_{1}(X)$ C.

Let us consider $\mathbf{C} \in \mathbb{P}^{2}$. We consider $\operatorname{Sing}(\mathbf{C})$ the set of singular points of

Let $\sigma: Y \rightarrow \mathbb{P}^{2}$ the minimal embedded resolution of the singular points of C. We set

$$
D:=\sigma^{*} \mathbf{C}=C_{1}+\cdots+C_{r}+\sum_{P \in \operatorname{Sing}(\mathbf{C})} \sum_{j=1}^{l_{p}} m_{j}^{P} E_{j}^{P},
$$

the total transform of $\mathbf{C}$ where $C_{1}, \ldots, C_{r}$ are the irreducible components of the strict transform of $\mathbf{C}$ and $E_{1}^{P}, \ldots, E_{l_{p}}^{P}$ are the irreducible components of $E_{P}:=\sigma^{-1}(P)$, which is the exceptional locus of $\sigma$ at $P$, for each $P \in \operatorname{Sing}(\mathbf{C})$. No smooth point is blown up and then, the multiplicities $m_{j}^{P}$ are at least two.

We consider $\mathscr{O}(1)$, the rank one, locally free sheaf on $\mathbb{P}^{2}$ whose Chern class is 1 ; we consider $\mathscr{O}(1)=\mathscr{O}(H)$ where $H$ is a line in $\mathbb{P}^{2}$ in general position with respect to $\mathrm{C}$. We identify $H$ with $\sigma^{*}(H) . \mathscr{L}:=\sigma^{*} \mathscr{O}(1)$ is a sheaf on $Y$ and $\mathscr{L}=\mathscr{O}(H)$.

2.1. Definition. The $k$ th sheaf associated to $\mathbf{C}, k=0, \ldots, N-1$, is the sheaf on $Y$ defined by

$$
\mathscr{L}^{(k)}:=\mathscr{L}^{k} \otimes \mathscr{O}\left(-\sum_{P, j}\left[\frac{m_{j}^{P} k}{N}\right] E_{j}^{P}\right) .
$$
$X$.

The next result of [E] uses the Mixed Hodge Structure on the cohomology of

2.2. Proposition. Let $H^{1}(X ; \mathbb{C}):=\bigoplus_{k=0}^{N-1} H^{k}$ be the decomposition of $H^{1}(X ; \mathbb{C})$ in eigenspaces for the action of the monodromy of the covering $\tau$, where the eigenvalue of $H^{k}$ is $\exp (2 i \pi k / N)$. Then, $\operatorname{dim} H^{0}=r-1$ and $H^{k}$ is naturally isomorphic to $H^{1}\left(Y, \mathscr{L}^{(k)}\right) \oplus \overline{H^{1}\left(Y, \mathscr{L}^{(N-k)}\right)}$, for $k=1, \ldots, N-1$.

2.3. Using the adjunction formula [BPV, Chapter I], the canonical sheaf of $Y$ may be presented as

$$
\mathscr{K}_{Y}=\mathscr{O}\left(K_{Y}\right)=\mathscr{O}\left(-3 H+\sum_{P} \sum_{j} \kappa_{j}^{P} E_{j}^{P}\right)
$$

where $\kappa_{j}^{P}$ are the only solution of

$$
E_{j}^{P} \cdot\left(\sum_{l} \lambda_{l}^{P} E_{l}^{P}\right)=-2-E_{j}^{P} \cdot E_{j}^{P} .
$$


2.4. Definition. Let us fix $P \in \operatorname{Sing}(\mathbf{C})$ and $k \in\{1, \ldots, N-1\}$. We define as $\mathscr{I}_{P, k, N}$ the ideal of $\mathscr{O}_{\mathbb{P}^{2}, P}$ whose elements are the germs $h$ of holomorphic functions at $P$ which can be lifted by $\sigma$ as sections of $\mathscr{O}\left(\sum\left(\kappa_{j}^{P}-\left[m_{j}^{P} k / N\right] E_{j}^{P}\right)\right)$ in the neighbourhood of the exceptional locus of $\sigma$ over $P$.

We define a homomorphism $\sigma_{k}: H^{0}\left(\mathbb{P}^{2}, \mathscr{O}(k-3)\right) \rightarrow \sum_{P \in \operatorname{Sing}(\mathbf{C})} \mathscr{O}_{\mathbb{P}^{2}, P} / \mathscr{I}_{P, k, N}$ as follows: Let $X, Y, Z$ be homogenous coordinates of $\mathbb{P}^{2}$ such that $Z=0$ is the equation for $L$. Then, the domain of $\sigma_{k}$ is identified with the set of polynomials in $X, Y$ of degree not bigger than $k-3$. The image of a polynomial $g$ is the formal sum of the germs of $g$ at $P, P \in \operatorname{Sing}(\mathbf{C})$, regarded as elements of the quotient rings.

The kernel of $\sigma_{k}$ is the set of equations of plane projective curves of degree $k-3$ which pass through the points of $\operatorname{Sing}(\mathbf{C})$ following the instructions given by the ideals $\mathscr{I}_{P, k, N}$. These instructions impose conditions on the Enriques' infinitely near points of the curves. The next formula is proved in [A].

2.5. Theorem. The dimension of $H^{k}$ is $\sum_{P \in \operatorname{Sing}(\mathbf{C})} d_{k}^{P}-((k-1)(k-2) / 2+$ $\operatorname{dim} \operatorname{ker} \sigma_{k}$ ) where

$$
d_{k}^{P}=\frac{1}{2}\left(\sum_{a \in S(P)}\left[\frac{m_{a} k}{d}\right] E_{a}\right) \cdot\left(\sum_{a \in S(P)}\left(\kappa_{a}-\left[\frac{m_{a} k}{d}\right]\right) E_{a}\right) .
$$

In fact, $\operatorname{dim} H^{k} \leq \sum_{P \in \operatorname{Sing}(\mathbf{C})} d_{k}^{P}$.

In the next section we will use this formula with two annihilation theorems.

2.6. Theorem [Z2]. If $\mathbf{C}$ is irreducible and $N$ is a prime power, then $H^{1}(X, \mathbb{C})$ $=0$.

2.7. Proposition [E]. Let $k \in\{1, \ldots, N-1\}$; let us suppose that $m_{j}^{P} k / N$ is never an integer number. Then, $H^{k}=0$.

This last result is more general than Esnault's result but the proof is essentially the same.

\section{EXAMPLES AND HYPERPLANE ARRANGEMENTS}

3.1. Example. There is no curve of degree 7 and 11 cusps in the singular locus.

For a cusp, the multiplicities in the exceptional divisor are 2, 3, 6. Because of Proposition 2.7, $b_{1}(X)=r-1$. Nevertheless, if we compute the coefficients $a_{j}^{P}$ of the canonical divisor, we get $1,2,4$, for each $P$. We find that $d_{6}^{P}=1$ for each $P$. As $\operatorname{dim} H^{0}\left(\mathbb{P}^{2}, \mathscr{O}(3)\right)=10$, we have that $b_{1}(X)>r-1$, and then, a contradiction.

3.2. Example. There is no curve of degree 8 and 16 cusps in the singular locus.

As in example 3.5 we see that $d_{7}=16$. Because $\operatorname{dim} H^{0}\left(\mathbb{P}^{2}, \mathscr{O}(4)\right)=15$, we have that $b_{1}(X)>r-1$. We also have $d_{k}=0$ if $k<7$; as 7 is prime to 2,3 , and 6, it implies that $b_{1}(X)=r-1$ because of Proposition 2.7, a contradiction.

From now on, we are only interested in curves $\mathbf{C}$, having only ordinary singular points. That is to say, if $P \in \operatorname{Sing}(\mathbf{C}), \mathbf{C}$ has $m_{P}$ pairwise transversal smooth local components at $P$. The surface $Y$ is obtained by blowing up the points $P \in \operatorname{Sing}(\mathbf{C})$. Then, the exceptional divisor of $\sigma$ has \# $\operatorname{Sing}(\mathbf{C})$ pairwise 
disjoint irreducible components, denoted by $E_{P}$; they are projective lines with self-intersection -1 and multiplicity $m_{P}$. In this case, the divisors $D$ and $K_{Y}$ of last paragraph are

$$
D=C_{1}+\cdots+C_{r}+\sum_{P \in \operatorname{Sing}(\mathbf{C})} m_{P} E_{P}, \quad K_{Y}=-3 L+\sum_{P \in \operatorname{Sing}(\mathbf{C})} E_{P} .
$$

For $k$ and $P$, the divisor involved in the definition of $\mathscr{I}_{P, k, N}$ is $\left(1-\left[m_{p} k / N\right]\right) E_{P}$.

Using local coordinates at $P \in \operatorname{Sing}(\mathbf{C})$ we see $\mathscr{I}_{P, k, N}=\mathscr{M}_{P}^{\left[m_{p} k / N\right]-1}$, where $\mathscr{M}_{P}$ is the maximal ideal of $\mathscr{O}_{\mathbb{P}^{2}, P}$.

We remark that

$$
\operatorname{dim} \mathscr{O}_{\mathbb{P}^{2}, P} / \mathscr{I}_{P, k, N}=\sum \frac{\left[m_{P} k / N\right]\left(\left[m_{P} k / N\right]-1\right)}{2}=d_{k}^{P} .
$$

Therefore,

$$
b_{1}(X)=r-1+2 \sum_{k=1}^{N-1} \operatorname{dim} \operatorname{coker} \sigma_{k} \text {, }
$$

where

$$
\sigma_{k}: H^{0}\left(\mathbb{P}^{2}, \mathscr{O}(k-3)\right) \rightarrow \sum_{P \in \operatorname{Sing}(\mathbf{C})} \frac{\mathscr{O}_{\mathbb{P}^{2}, P}}{\mathscr{M}_{P}^{[m p k / N]-1}}
$$

3.3. Remark. The kernel of $\sigma_{k}$ is the set of equations of the curves of degree $k-3$ passing through $P \in \operatorname{Sing}(\mathbf{C})$ with multiplicity at least $m_{P}$.

We are going to apply these results to line arrangements. We can find most definitions and results in [O]. A line arrangement is a curve $\mathbf{C}$ of degree $N$ which decomposes into $N$ different lines. We want to know which topological invariants of the couple $\left(\mathbb{P}^{2}, \mathbf{C}\right)$ only depend on the combinatorics of the arrangement, i.e., how the $N$ lines do intersect.

The cohomology of the complement $E$ of $\mathbf{C}$ is well known; $b_{1}(E)=N-1$ is a consequence of the following general result:

3.4. Lemma. Let $\mathbf{C}_{1}, \ldots, \mathbf{C}_{r}$ irreducible curves of degrees $d_{1}, \ldots, d_{r}$. Then

$$
H^{1}\left(\mathbb{P}^{2} \backslash \bigcup_{i=1}^{r} \mathbf{C}_{i} ; \mathbb{Z}\right)=\left|\mu_{1}, \ldots, \mu_{r}: d_{1} \cdot \mu_{1}+\cdots+d_{r} \cdot \mu_{r}=0\right| \text {. }
$$

In this case it is easy to calculate $b_{2}(E)$ using the facts that the Milnor number of an ordinary singularity of multiplicity $m$ is $\mu=(m-1)^{2}$ and $\chi(E)=N^{2}-3 N+3-\sum_{P \in \operatorname{Sing}(\mathbf{C})} \mu_{P}$.

The results of the last paragraph give some new information about $X$; this space is not well known. These results allow us to give some counterexamples to the conjecture stated in the introduction.

3.5. Example. Let $\mathbf{C}_{N}$ be an arrangement of $N$ lines passing through the point $[0: 0: 1]$. We have

$$
\sigma_{k}: H^{0}\left(\mathbb{P}^{2}, \mathscr{O}(k-3)\right) \rightarrow \mathbb{C}\{x, y\} /(x, y)^{k-1} .
$$

This map is one-to-one, as a curve of degree $k-3$ cannot have points of multiplicity $k-1$. Then

$$
\operatorname{dim} \operatorname{coker} \sigma_{k}=\frac{k(k-1)}{2}-\frac{(k-1)(k-2)}{2}=k-1 .
$$




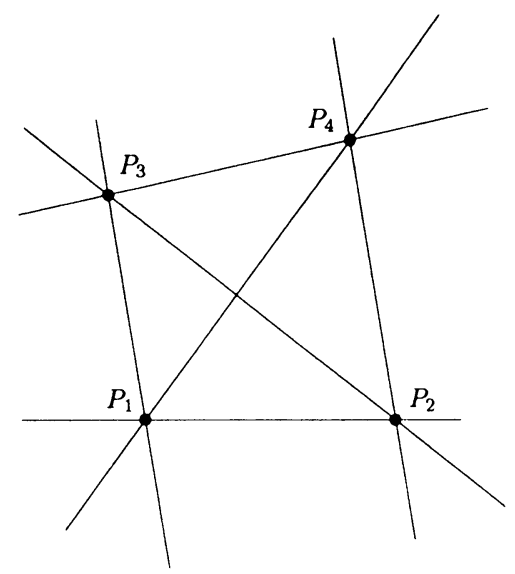

FIGURE 3.1

We have just seen that the monodromy does not act trivially on $H^{1}(X, \mathbf{C})$, and

$$
b_{1}(X)=N-1+2 \sum_{k=1}^{N-1}(k-1)=(N-1)^{2} \text {. }
$$

3.6. Example. Let us consider the arrangement of lines (Figure 3.1) passing through points $P_{1}, \ldots, P_{4}$, such that no three of them are in a line. There are four triple points. We are only interested in $\sigma_{4}$ :

$$
\sigma_{4}: H^{0}\left(\mathbb{P}^{2}, \mathscr{O}(1)\right) \rightarrow \sum_{i=1}^{4} \frac{\mathscr{O}_{P_{i}}}{\mathscr{M}_{P_{i}}}
$$

The kernel of this map is zero: $\operatorname{dim} \operatorname{coker} \sigma_{4}=1$, and therefore

$$
b_{1}(X)=6-1+2 \cdot 1=7 \text {. }
$$

One open problem in this theory is to know whether the topology of the Milnor fiber $X$ depends only on the combinatorics. It is not known for the Betti numbers of $X$, though the answer is yes for the Betti numbers of the complement $E$; it is also known that the ring $H^{*}(E ; \mathbb{C})$ is a combinatorial invariant (see [O]).

We are going to consider two examples of arrangements with not very different combinatorics; the Betti numbers of $E$ are the same, but it is not the case for the Betti numbers of $X$.

Both arrangements have nine lines, nine double points, nine triple points, and each line contains exactly three triple points. In both cases, $b_{1}(E)=8$. On the other hand, $b_{1}(X)=8+2 \operatorname{dim}$ coker $\sigma_{6}$, with

$$
\sigma_{6}: H^{0}\left(\mathbb{P}^{2}, \mathscr{O}(3)\right) \rightarrow \sum_{i=1}^{9} \frac{\mathscr{O}_{P_{i}}}{\mathscr{M}_{P_{i}}}
$$

We must find the cubics passing through the nine triple points. 
3.7. Example. There exists an arrangement as above such that $b_{1}(X)=10$.

Proof. There are three cubics $Q_{1}, Q_{2}, Q_{3}$ where each $Q_{i}$ decomposes in three lines in general position. The nine lines are pairwise different and the three cubics have exactly nine common points. The arrangement $Q_{1} \cup Q_{2} \cup Q_{3}$ is the good one: $Q_{1}$ and $Q_{2}$ generate the set of cubics passing through the nine triple points. Then, $\operatorname{dim}$ coker $\sigma_{6}=1$ and $b_{1}(X)=8+2=10$.

Such an arrangement exists:

$$
\begin{aligned}
& a(a+1) \neq 0, \\
& Q_{1}=X Z(a b X+(a-a b+1) Y+b Z), \quad b(b-1) \neq 0, \\
& Q_{2}=Y(X-Y-Z)(a X+Y+b Z), \quad a-a b+1 \neq 0, \\
& Q_{3}=(X-Y)(a X+Y+Z)(Y+b Z), \quad a+b \neq 0, \\
& a b+b-1 \neq 0 \text {. }
\end{aligned}
$$

We have the equation of the three cubics.

3.8. Example. There exists an arrangement as above such that $b_{1}(X)=8$.

Proof. We give the equations of an arrangement whose triple points determine one cubic; then, $\operatorname{dim}$ coker $\sigma_{6}=0$ and $b_{1}(X)=8$. The existence of only one cubic guarantees that the combinatorics of this arrangement and that of the arrangement in Example 3.7 are different. The example is

$$
\begin{array}{rr}
Q=X(X+Y)(c X+(c+1) Y+b Z) & a b c \neq 0, \\
Y(X+a Z)(c(a+b) X+a(c+1) Y+a b(c+1) Z) & c+1 \neq 0, \\
Z(Y+b Z)(c X+(c+1) Y+a c Z), & a c-b \neq 0, \\
a+b & \neq 0, \\
& c(a-b c)-b(c+1)=0 .
\end{array}
$$

These equations give the end of the proof.

\section{REFERENCES}

[A] E. Artal, Les couples de Zariski, J. Algebraic Geom. (to appear).

[BPV] W. Barth, C. Peters, and A. Van de Ven, Compact complex surfaces, Springer-Verlag, Berlin, Heidelberg, and New York, 1984.

[E] H. Esnault, Fibre de Milnor d'un cône sur une courbe algébrique plane, Invent. Math. 68 (1982), 477-496.

[O] P. Orlik, Introduction to arrangements, Proc. Sympos. Pure Math., vol. 72, Amer. Math. Soc., Providence, RI, 1989.

[Z1] O. Zariski, On the linear connection index of the algebraic surfaces, Proc. Nat. Acad. Sci. U.S.A. 15 (1929), 494-501.

[Z2] _ On the irregularity of cyclic multiple planes, Ann. of Math. (2) 32 (1931), 485-511.

Departamento de Matemáticas, Universidad de Zaragoza, E-50009 Zaragoza, Spain Current address: Departamento de Geometría y Topología, Universidad Complutense, E-28040 Madrid, Spain

E-mail address: artal@mat.ucm.es 